\title{
On the Path from the Turbulent Vortex Dynamo Theory to Diagnosis of Tropical Cyclogenesis
}

\author{
Galina Levina \\ Space Research Institute, Russian Academy of Sciences, Moscow, Russia \\ Email: levina@iki.rssi.ru
}

How to cite this paper: Levina, G. (2018) On the Path from the Turbulent Vortex Dynamo Theory to Diagnosis of Tropical Cyclogenesis. Open Journal of Fluid Dynamics, 8, 86-114.

https://doi.org/10.4236/ojfd.2018.81008

Received: February 12, 2018

Accepted: March 26, 2018

Published: March 29, 2018

Copyright $\odot 2018$ by author and Scientific Research Publishing Inc. This work is licensed under the Creative Commons Attribution International License (CC BY 4.0).

http://creativecommons.org/licenses/by/4.0/

\begin{abstract}
An overview of researches is presented, which was focused on application of a theoretical hypothesis on the turbulent vortex dynamo to the study of tropical cyclogenesis. The dynamo effect is related to the special properties of small-scale helical turbulence with the broken mirror symmetry and was hypothesized to result in large-scale vortices generation in both hydrodynamic and atmospheric turbulence. To introduce this abstract theory into tropical cyclone research, a recent discovery of vortical moist convection in the tropics is emphasized. Based on this finding, we discuss and substantiate the crucial role of rotating cumulonimbus clouds, known as vortical hot towers (VHTs), as a necessary element to provide the dynamo effect. An analogy is traced between the role of interaction "moist convection-vertical wind shear" in creating the vortex dynamo in the atmosphere and the role of the mean electromotive force providing the MHD dynamo in electrically conducting medium. Throughout the review of novel results, a pivotal role of the Russian-American collaboration on examining a helical self-organization of moist convective atmospheric turbulence under tropical cyclone formation by use of cloud-resolving numerical simulation is accented. The efforts resulted in application of the vortex dynamo theory to diagnose a time when cyclogenesis commences in a favorable tropical environment. This may help elaborate a universally accepted definition of tropical cyclogenesis that currently does not exist and contribute to practical purposes of diagnosis and forecasting.
\end{abstract}

\section{Keywords}

Large-Scale Helical-Vortex Instability, Tropical Cyclone Formation, Rotating Cumulus Convection, Cloud-Resolving Numerical Analysis

\section{Introduction}

The present review-research article is aimed at bridging the fundamentals of the 
theory of turbulence and the most advanced atmospheric studies based on cloud-resolving numerical simulation in order to approach unraveling the awesome natural phenomenon of tropical cyclogenesis.

Incipient research efforts towards this goal are dating back to the early 1980s, when almost simultaneously, there appeared two publications [1] [2]. In the first of them, the theory of turbulent vortex dynamo was put forward, and in the second one, the possibility of applying this theory to explain the formation of tropical cyclones in the Earth's atmosphere was considered. The vortex dynamo mechanism [1] is based on some intrinsic peculiarities of the small-scale helical turbulence of the velocity field [3] [4] [5] [6], characterized by the broken mirror symmetry. Under certain conditions, the helical turbulence is capable of amplifying and sustaining large-scale vortex perturbations by transferring energy from small scales to large ones and providing an inverse energy cascade. A featured characteristic of the new large-scale, helical-vortex, instability found in [1] was the existence of an excitation threshold of instability. It should be noted that the excitation of threshold kind is also inherent in other large-scale instabilities found in turbulent media with symmetry breaking: the alpha effect in magnetohydrodynamics [7] [8] [9] and anisotropic kinetic alpha effect (AKA-effect) in an electrically non-conducting medium [10]. In the atmosphere-oriented work [2], formulas were obtained for the critical values of a number of parameters that determine the emergence of large-scale helical-vortex instability, and on their basis, quantitative estimates were calculated for the conditions in the Earth's atmosphere, which are corresponded to the formation of tropical cyclones. Later, the theoretical formulas [2] were also applied to obtain quantitative estimates for the atmosphere of Jupiter. This made it possible to adequately interpret the dimensions and lifetime of large-scale long-lived vortex disturbances caused by the fall of the fragments of comet Shoemaker-Levy 9 in 1994 [11] [12]. Both applications to the atmospheres of planets, the Earth and Jupiter, were discussed in detail in the review [13].

Following the publications [1] [2] and inspired by their findings, a wide-ranging research program headed by the Space Research Institute and aimed at testing the hypothesis on the turbulent vortex dynamo was initiated in several scientific centers across the USSR. This included theoretical, experimental, and numerical modeling of developed turbulent convection in rotating non-uniformly heated fluids with a focus on conditions and ways of generation of intense large-scale spiral vortex structures and was targeted at peculiarities of tropical cyclones formation in the Earth's atmosphere [13]. A close attention was also paid to a search for ways of controlling the formation of such structures. Undertaken theoretical analysis resulted in a few ideas of how the formation of intense large-scale vortices might be affected, which were offered for testing in the appropriately designed laboratory experiments [13]. In the context of our current discussion it is worth to recall the one [14], which was connected with introducing of external small-scale helicity into a rotating layer of non-uniformly heated 
fluid in order to initiate or intensify an already existing large-scale spiral vortex or, alternatively, to suppress its amplification or formation altogether. The program was progressing successfully and culminated in two field experiments in the tropical Pacific-expeditions "Typhoon-89" and "Typhoon-90" of 100 days each, on the board of one and four research ships in typhoons' seasons of 1989 and 1990, respectively. During the expeditions, the emphasis was on measurements that would make it possible to reveal any signs indicating the action of the vortex dynamo in pre-typhoon states of the tropical atmosphere. Spectra of atmospheric turbulence were obtained and examined for various synoptic conditions, which included: the regions without any visible signs of cyclonic or anticyclonic circulation; near the centers of tropical depressions (cyclonic vortices with maximum sustained tangential near-surface winds up to $17 \mathrm{~m} / \mathrm{s}$ ), which were rapidly deepening and intensifying into tropical storms (maximum winds were within $18-32 \mathrm{~m} / \mathrm{s}$ ) during the observations; on the periphery of a developed tropical cyclone (maximum winds $33 \mathrm{~m} / \mathrm{s}$ or higher). The analysis carried out found a non-zero helicity of the atmospheric turbulence and gave an implication that inverse energy transfer might exist from the small scales to large ones in conditions of tropical cyclone formation [15]. The program was stopped after the collapse of the Soviet Union in 1991. However, during the next decade, some investigations were continued at the Space Research Institute in Moscow and Institute of Continuous Media Mechanics in Perm. The achievements of the 20th century on the turbulent vortex dynamo and related topics were summed in the review [13]

The present work is a continuation of the efforts discussed above. In order to introduce the hypothesis on the turbulent vortex dynamo into tropical cyclone research, a recent discovery of vortical moist convection in the tropics is emphasized. Based on this finding, we discuss and substantiate the crucial role of rotating cumulonimbus clouds, known as vortical hot towers (VHTs), as a necessary element to provide the dynamo effect in conditions of tropical cyclone formation. Based on the mathematical model of the turbulent vortex dynamo in a convective system [13] and results of numerical simulation carried out for this model, an analogy is traced between the role of interaction "moist convection-vertical wind shear" (described by a hypothetical "vortex-motive" force in the model) in creating the vortex dynamo in the atmosphere and the role of the mean electromotive force providing the magnetohydrodynamic (MHD) dynamo in electrically conducting medium. Bearing in mind an existence of excitation threshold for the large-scale helical-vortex instability predicted within the theoretical model and confirmed by numerical analysis for this model, we propose introducing quantitative criteria, which take into account helicity, convective available potential energy (CAPE), and vertical wind shear (similarly to those applied for midlatitude severe storms), in order to use them for study and diagnosis of tropical cyclogenesis.

The paper is organized as follows. In Section 2, we discuss the mathematical 
model of the turbulent vortex dynamo in a rotating non-uniformly heated fluid; a numerical approach designed to study the large-scale helical-vortex instability is presented and results of numerical investigation of the model are summarized. Section 3 highlights the discovery of vortical moist convection in the tropics and its helical features. In Section 4, we describe the implementation of post-processing of atmospheric simulation data and examine the process of helicity generation on cloud- and mesoscales. Section 5 presents a mechanism of a VHT formation and helicity generation through interaction between a convective updraft and vertical wind shear. A crucial role of VHTs is emphasized in providing a special topology of a forming mesoscale vortex, which is characterized by the linkage of the primary (tangential) and secondary (transverse) circulation. Based on it, a rationale is proposed for how VHTs can participate ensuring the action of the turbulent vortex dynamo in the tropical atmosphere. Section 6 presents how the concept of vortex dynamo can be applied for practical purposes of diagnosing the commencement time of tropical cyclogenesis. In Section 7 "Conclusions", we summarize the main findings and offer a perspective for these investigations.

\section{Turbulent Vortex Dynamo in a Convective System}

A fundamental hypothesis is known in the theory of turbulence that an inverse energy transfer from small to large scales is possible in the helical turbulent medium with the broken mirror symmetry [5]. This may result in a large-scale instability leading to the formation of organized structures with space- and time-scales essentially exceeding those of background turbulence. The confirmation to the above hypothesis was first discovered in magnetohydrodynamics [8]. This phenomenon, known as the alpha effect, allows us to explain the growth of large-scale magnetic fields in electrically conducting medium and forms the basis of the MHD dynamo theory [7] [8] [9]. The formal similarity of equations describing the magnetic field in a moving electrically conducting medium and vorticity field in non-conducting fluids [6] gave an impetus to a search for analogs to this phenomenon in general hydrodynamics. The first theoretical example of large-scale helical instability in general (non-MHD) hydrodynamics was found in [1] and, by analogy, coined the hydrodynamic alpha-effect.

A mathematical model of the turbulent vortex dynamo in a convectively unstable non-uniformly heated fluid was first proposed in [16] and was obtained by the same methods as the model in [1] without a temperature field, namely, by use of the mean-field theory [9]. Further development and generalization of this model was performed in [17]-[22], in which the problem formulations were brought closer to the real conditions of tropical cyclones formation by taking into account the rotation of the atmospheric layer and phase transitions of moisture. In [21] [22], the method of multiscale asymptotic expansions was applied, which allows the principal order in which the instability emerges to be discerned from the entire hierarchy of perturbations. The findings of investigations [19] [20] emphasized the role of the joint effect of the Coriolis force and 
the temperature profile nonlinearity provided by internal heat release due to water vapor condensation in order to reach generation of the non-zero helicity of thermoconvective turbulence, and as a result, the dynamo-effect. Thus, being in good agreement with earlier works [7] [23] about the mutual role of buoyancy and Coriolis forces in generating helicity, the authors [13] [19] [20] contributed by highlighting the role of internal energy sources in this process. Independently of [19] [20], processes of vorticity and helicity generation in the moist rotating turbulent atmosphere were theoretically examined in [24] [25], in which the similar result was accented, namely, an energy release due to phase transition of moisture was required to reach the non-zero dynamo effect.

\subsection{Helicity of the Velocity Field}

Helicity of the velocity field is a pseudoscalar quantity defined as the scalar product of velocity $\boldsymbol{v}(\boldsymbol{r}, t)$ and vorticity $\boldsymbol{\omega}(\boldsymbol{r}, t)=$ curl $\boldsymbol{v}$ vectors [3]. The volume integral calculated in a specific space domain,

$$
H=\int \boldsymbol{v} \cdot \boldsymbol{\omega} \mathrm{d} \boldsymbol{r},
$$

gives the helicity of vortex system, where $\boldsymbol{v} \cdot \boldsymbol{\omega}$ is the helicity density of the flow. Both quantities are pseudoscalars, i.e., they change sign under change from a right-handed to a left-handed frame of reference [4].

A non-vanishing helicity implies the symmetry break of turbulence with respect to coordinate system reflections [3] [4] [5] [6]. The mean helicity, like energy, is an inviscid quadratic constant of motion in barotropic fluids. If we choose a right-handed Cartesian or orthogonal curvilinear frame for our further consideration, positive mean helicity will be generated in the moist atmosphere under the predominance of cyclonic updrafts and/or anticyclonic downdraft motions. Similarly, negative helicity will be generated for the case of anticyclonic updrafts and/or cyclonic downdraft flows.

However, unlike energy the helicity can be both positive and negative. Its sign determines the predominance of the left-handed or the right-handed spiral motions in the examined flow.

Helicity is one of the most important characteristics for describing the structure of vortex fields. This quantity is a topological invariant, which measures the degree of linkage of the vortex lines [3] [4] [5] [6]. Let us only reproduce here a picture from Moffatt's seminal work [3] (Figure 1), as a simple illustration for this complex topological notion. In further analysis of helical atmospheric flows, this may help imagine-how do they look?

The sources of helical turbulence are known to be the force fields of a pseudovector nature, such as magnetic or Coriolis force fields.

\subsection{Mean-Field Equations}

Let us analyze and discuss the mathematical dynamo-model for the convective 


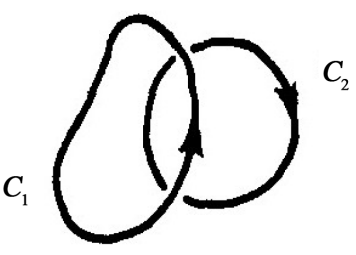

(a)

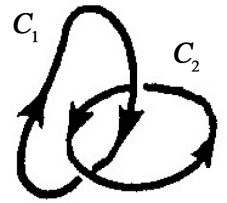

(b)

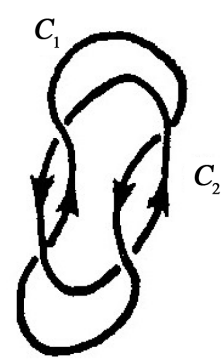

(c)

Figure 1. The degree of linkage of two closed filaments $C_{1}, C_{2}$ (where $\alpha_{12}$ is the "winding number" of the curves $C_{1}$ and $C_{2}$ ). The choice of sign in (b); (c) is determined by the relative orientation of the two filaments. Borrowed from [3]. (a) $\alpha_{12}=0$; (b) $\alpha_{12}=-1$; (c) $\alpha_{12}=$ 2.

system in more detail.

The most demonstrative physical interpretation of the obtained dynamo-effect can be given in terms of toroidal and poloidal component of the vector velocity field, i.e. in the form of representation that is frequently used in magnetohydrodynamics [7] [9] and is well suited for transformation of corresponding vector equations to the system of equations for scalar functions. Let us choose $\boldsymbol{V}$ for denoting the mean velocity field $\left\langle v_{i}\right\rangle$ and express it in the following form:

$$
\boldsymbol{V}=\boldsymbol{V}_{\mathrm{T}}+\boldsymbol{V}_{\mathrm{P}}, \boldsymbol{V}_{\mathrm{T}}=\operatorname{curl}(e \psi), \boldsymbol{V}_{\mathrm{P}}=\operatorname{curl} \operatorname{curl}(\boldsymbol{e} \varphi), \boldsymbol{e}=\{0,0,1\} .
$$

Bearing in mind the formation of tropical cyclones in the atmosphere as well as vortex flows in rotating non-uniformly heated fluids, we can also interpret this representation in common terms of tangential and transverse (or overturning) circulation, respectively, in order to apply them in our further discussion.

In terms of expressions (2), a mathematical model of the turbulent vortex dynamo in a rotating horizontal layer of incompressible non-uniformly heated fluid can be written in the dimensionless form [13] [18] [19] [20] [26] [27]

$$
\begin{aligned}
& \left(\operatorname{Pr} \frac{\partial}{\partial t}-\Delta\right) T=-\Delta_{\perp} \varphi, \\
& \left(\frac{\partial}{\partial t}-\Delta\right) \Delta \varphi=R a T+C\left[(\boldsymbol{e} \cdot \nabla)^{2}-\Delta_{\perp}\right] \psi-T a^{1 / 2} \frac{\partial \psi}{\partial z}, \\
& \left(\frac{\partial}{\partial t}-\Delta\right) \psi=-C(\boldsymbol{e} \cdot \nabla)^{2} \varphi+T a^{1 / 2} \frac{\partial \varphi}{\partial z}, \\
& \operatorname{Pr}=\frac{v}{\chi}, \quad R a=\frac{g \beta A h^{4}}{v \chi}, \quad C \propto \Omega \Lambda, T a=\frac{4 \Omega^{2} h^{4}}{v^{2}} .
\end{aligned}
$$

Here, $T$ is the temperature, $\varphi$ and $\psi$ are the poloidal and toroidal potentials of the velocity field, and $\Delta_{\perp}=\partial^{2} / \partial x^{2}+\partial^{2} / \partial y^{2}$ is the two-dimensional Laplace operator. Pr, Ra, and Ta are the dimensionless Prandtl, Rayleigh, and Taylor numbers, $\boldsymbol{e}$ the unit vector directed vertically upward, $A$ the uniform temperature gradient between the horizontal boundaries of the layer, $g$ the gravity acceleration, $\beta$ the coefficient of thermal expansion, $h$ the layer height. The dimen- 
sionless parameter $C$ characterizing the small-scale turbulence is related in a rather complicated manner to the turbulence characteristics such as the most energetic scale $\lambda$ and characteristic time $\tau$ of the turbulent velocity correlation [19] [26] [27], and is proportional to the angular velocity of a fluid layer rotation $\Omega$, and the power of internal heat sources $\Lambda$.

The system of dynamic linear Equations (3) for three large-scale fields includes two different positive feedbacks. One of them acts between the poloidal $\varphi$-component of the velocity field and the field of temperature disturbance $T$. It links the first and the second equations in system (3) and leads to natural convective instability. The other directly links the components $\varphi$ and $\psi$ of the velocity field, i.e. the second and the third equations from system (3). Here it is important to note that, as applied to tropical cyclones, this would imply an immediate positive feedback between the tangential and overturning circulation. This feedback, being generated by special properties of small-scale helical turbulence and, therefore, named the helical feedback [13] [26] [27], is maintained only through the parameter $C$, whose explicit form was first given in [19] and discussed in detail in the above works.

As we can see in model (3), in the absence of volumetric heat release $(\Lambda=0)$, the coefficient $C=0$ and, accordingly, the corresponding terms in the equations vanish, i.e. the effect of the vortex dynamo is impossible. As applied to the atmosphere, this means that heating the layer from below owing solar radiation received by the underlying surface is insufficient for the occurrence of large-scale instability.

If $C \neq 0$, then the mean large-scale flow $V$ shows a special topological property - the linkage of vortex lines of poloidal and toroidal flow component [3] [4] [5] [6].

Thus, the positive helical feedback is responsible for a new type of instability, namely, the large-scale helical-vortex instability [13]. In this meaning, $C$-terms in model (3) are analogous to the mean electromotive force [7] [8] [9] providing the generation of large-scale magnetic fields in magnetohydrodynamics.

\subsection{A "Vortex-Motive" Force}

Unlike the alpha effect in an electrically conducting medium based on the interaction of two different physical fields-the magnetic field and the velocity field-in its hydrodynamic analog, the dynamo effect is to be provided only by the singularities of a single velocity field. In magnetohydrodynamics, the mean electromotive force [7] [8] [9], linking the two fields, ensures the amplification and maintenance of large-scale magnetic field. The realization of this phenomenon can be well illustrated in specific cases, for example, for the generation of large-scale magnetic fields of astrophysical objects.

The vortex dynamo should operate by linking the poloidal and toroidal component of the velocity field. In mathematical model (3), $C$-terms responsible for the positive feedback can also be represented as a force of some kind [13] [26] 
[27], which, by analogy, could be called the "vortex-motive" force

$$
\boldsymbol{f}=C\left\{\boldsymbol{e} \cdot(\operatorname{curl} \boldsymbol{V})_{z}-\partial(\boldsymbol{e} \times \boldsymbol{V}) / \partial z\right\}, \boldsymbol{e}=\{0,0,1\} .
$$

It seems both curious and useful to give an explicit representation of components of such hypothetical vortex-motive force

$$
\boldsymbol{f}=\left\{\frac{\partial v}{\partial z},-\frac{\partial u}{\partial z}, \frac{\partial v}{\partial x}-\frac{\partial u}{\partial y}\right\} .
$$

The first two terms in Formula (5) describe the vertical shear of horizontal velocity whilst the third one is the vertical component of vorticity. As our analysis showed [26] [27], the third term is of paramount importance because of its crucial role in the closing of the positive feedback loop between the poloidal and toroidal field.

The emergence of positive helical feedback between the toroidal and poloidal component of the velocity field can be considered as a clear sign indicating the onset of helical-vortex instability. This served a basic idea for developing a numerical approach [26] [27] that had at first been applied to simulate and thoroughly examine the evolution of helical-vortex instability in Rayleigh-Bénard convection and later, it was adapted for diagnosing the commencement of tropical cyclogenesis in the atmosphere.

\subsection{Numerical Approach for Studying the Large-Scale Helical-Vortex Instability}

A key to the approach [26] [27] was the addition of a model force to the convection equations. It is widespread in turbulence modeling to have a forcing function driving a turbulent flow. However, the forcing term in simulations [26] [27] was not assigned to be a driving force. It was applied to naturally induced, fully developed convective flows and was only considered to be responsible for spiralizing the flow and generating the positive helical feedback. For this purpose, it was difficult to come up with anything better than the vortex-motive force defined by expression (4).

Numerical investigations carried out in [26] [27]:

- gave a vivid example of non-zero mean helicity, $\langle H\rangle \neq 0$, generation that implied the broken mirror symmetry,

- demonstrated new effects in flow structure and energetics attributed to a large-scale instability,

- confirmed a threshold type onset of this new helical instability,

- highlighted the generation of positive helical feedback between the tangential and overturning circulation in a vortex system,

- demonstrated how the helical feedback could be identified by examining integral kinetic energies of the tangential and overturning circulation,

- showed a probable scenario for development of the instability by merging of helical convective cells and consequent intensification of newly forming larger-scale helical vortices, 
- emphasized the crucial role of vertical flow component in the whole scenario of new instability,

- pointed out an increased effectiveness of heat transfer within a helical and larger vortex flow configuration.

These findings gave the authors of [26] [27] an impetus to search for possible application to tropical cyclone investigations.

\section{Helical Nature of Tropical Cyclogenesis}

The term "helical cyclogenesis" was first introduced in [28], in which a possible role of helicity fluctuations (when mean helicity vanished) was considered in providing an inverse energy cascade in three-dimensional turbulence. Later, an extended discussion was carried out in overview work [29] about how the proposed mechanism could hypothetically work enabling formation of mesoscale atmospheric phenomena such as tropical cyclones, subtropical hurricanes, and polar lows.

Despite the above mentioned long standing theoretical discussions on the role of helicity in tropical cyclogenesis, helical features of the velocity field have not been highlighted in real tropical cyclone investigations until very recently.

Probably, the hypothesis on the turbulent vortex dynamo [1] and, especially, its application to explain the formation of hurricane vortices in the atmosphere [2], both were somewhat ahead of their time. At that time, it was no awareness how the effect of the vortex dynamo could be implemented in the atmosphere.

In particular, in the case of tropical cyclones, the hypothetical realization of the vortex dynamo would mean the generation of positive helical feedback between the tangential and transverse circulation in a forming large-scale vortex, ensuring their mutual intensification. However, in nature, no vortex-motive force is known capable of providing the feedback and it was unclear whether such a scenario would be applicable to the atmosphere at all. To substantiate a feasibility of the vortex dynamo mechanism in the atmosphere, new knowledge about atmospheric processes was strongly needed.

\subsection{Vortical Moist Convection in the Tropics}

On the top of the third millennium, the knowledge was brought by high resolution three-dimensional numerical modeling of tropical cyclone formation. Pioneering near-cloud-resolving idealized simulations of tropical cyclogenesis under realistic meteorological conditions [30] [31] discovered and substantiated a vortical nature of atmospheric moist convection-vortical hot towers (VHTs). Moreover, what is of great importance, nearly simultaneously, such structures were first documented in observations by use of airborne Doppler radar data to show that VHTs were present in the genesis phase of Hurricane Dolly (1996) [32].

By now, the VHTs and their role in tropical cyclone formation have become a subject of significant amount of studies and reliably confirmed in observations 
performed by researchers over the world, see e.g. [33] [34] [35], and references therein. These rotating convective structures were closely investigated in several field experiments in the Atlantic and Pacific oceans, including an unprecedented trio of campaigns IFEX (the Intensity Forecasting Experiment)-GRIP (the Genesis and Rapid Intensification Processes)-PREDICT (the Pre-Depression Investigation of Cloud-Systems in the Tropics) in the Caribbean and West Atlantic, jointly organized by the National Oceanic and Atmospheric Administration (NOAA), the National Aeronautics and Space Administration (NASA), and the National Science Foundation (NSF) in late summer 2010 [34].

For the purpose of our further discussion, let us summarize briefly what has been known about these convective structures by now.

The cloud hot towers in the tropical atmosphere of the Earth (Figure 2(a)) were first described in 1958 [36] as horizontally small ( 10 - $30 \mathrm{~km}$ wide) but intense cumulonimbus convection cores that reached the tropopause, that in the tropics typically lies at least $15 \mathrm{~km}$ above sea level. The term "hot" comes not from the temperature of the air but because of the intense latent heat release due to phase transitions of moisture (vapor-water-ice) along the tower height.

The vortical tropical convection-VHTs-was first found nearly a half of the century later [30] [31] [32]. Typically, VHTs exhibit convective lifetimes on the order of one hour. For example, a thorough observational evidence of VHTs [37] showed that the specific updraft was $10 \mathrm{~km}$ wide and had vertical velocities reaching $10-25 \mathrm{~m} / \mathrm{s}$ above $6 \mathrm{~km}$. The convective cell was extending up to a height of $17 \mathrm{~km}$. The peak vertical velocity within this updraft exceeded $30 \mathrm{~m} / \mathrm{s}$. Maximum values of vertical relative vorticity reached up to $6-18 \times 10^{-3} 1 / \mathrm{s}$, what by the one-two order of magnitude exceeds the planetary rotation. The detailed evolution of the updraft observed during three successive fly-bys within approximately 40 minutes was presented in Figures 10-12 [37]. The essential physical characteristics of VHTs were highlighted in [38], where they were described as "deep moist convective clouds that rotate as an entity and/or contain
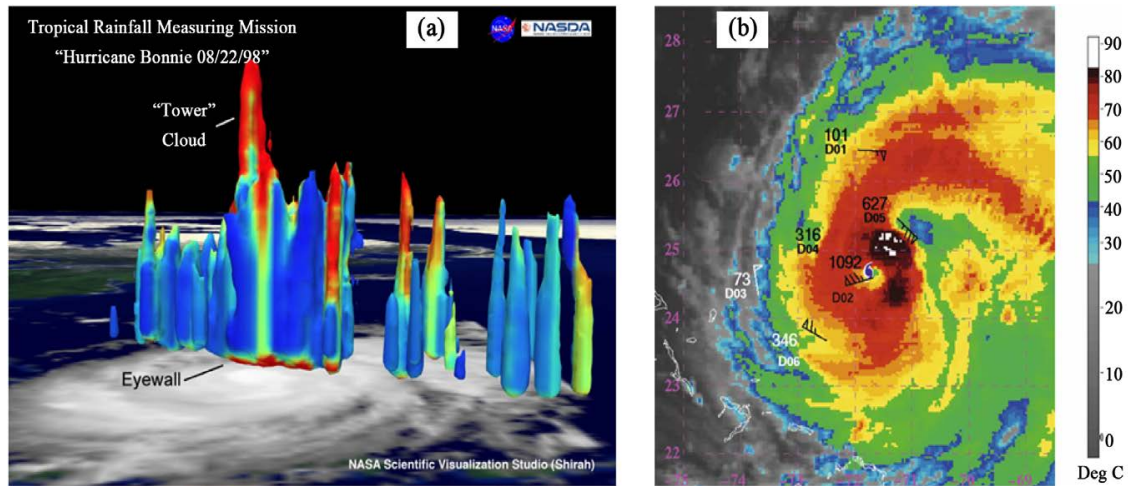

Figure 2. Hot towers in Hurricane Bonnie (1998). (a) NASA visualization for 22 August 1998 in a rapidly intensifying hurricane strength vortex (the altitude of towers is exaggerated) [41]; (b) Figure 4 [39]: Infrared satellite image at 2200 UTC 23 Aug, showing an intense local cell with a new cell forming upwind. 
updrafts that rotate in helical fashion (as in rotating Rayleigh-Bénard convection), ...These locally buoyant vortical plume structures amplify pre-existing cyclonic vorticity by at least an order of magnitude larger than that of the aggregate vortex." Finally, the authors [39] [40] paid the closest attention to the helical features of convective towers. In [39], helicity was calculated in Hurricane Bonnie (1998) using tropospheric-deep dropsonde soundings carried out by reconnaissance aircrafts during the NASA Convection and Moisture Experiment (CAMEX) and the most extreme values of helicity, among the largest ever reported in the literature, were found in the vicinity of deep convective cells. These cells reached as high as $17.5 \mathrm{~km}$. As it was noted in [40], in which helicity calculations were performed for eight tropical cyclones of 1998-2001 sampled during the CAMEX, VHTs are helical by definition because they contain coincident updrafts and vertical vorticity.

One of the most impressive visualizations of hot towers in a developed tropical cyclone was made by NASA (Figure 2(a)) and is available in Wikipedia [41]. Coincidentally, this turned out exactly to be for Hurricane Bonnie (1998).This allows us to confirm illustratively the above discussion that VHTs are helical. In Figure 2(b), for which the most consistent with time [41] figure was chosen from [39] (not with the highest helicity values), the color bar represents brightness temperature $\left({ }^{\circ} \mathrm{C}\right)$. Helicity values and mean winds over $0-6 \mathrm{~km}$ from sondes D1-D6 (released between 1845 and 2136 UTC 23 Aug) are also shown, plotted with respect to the moving center. These are shown in black or white depending on the background. Vertical wind shear was from the northwest at this time. The hurricane symbol represents the best-track center location. Thus, for tropical cyclones it has first been shown [39] that there exists an association of large helicity and intense convection.

\subsection{The Russian-American Collaboration}

By deepening and expanding the new knowledge gained about vortical convection [30] [32], the authors of [31] offered a new scenario of tropical cyclogenesis based on self-organization of convective processes in an otherwise favorable tropical environment. They considered the problem of the transformation of an isolated midlevel cyclonic mesoscale convective vortex (MCV) with weak cyclonic circulation at the ocean surface into a surface-concentrated (warm core) tropical depression vortex. The self-organization observed in their numerical simulation emphasized a key role of VHTs, and was realized via multiple convective structure mergers, which were accompanied by an upscale vorticity growth and system-scale convergence of absolute angular momentum about the nascent vortex's axis of circulation. It was showed [31], how, as a result of such flow evolution during about 24 hours, a surface-concentrated tropical depression vortex was formed on atmospheric mesoscale and intensified up to hurricane strength during 72 hours of experimental time.

The atmospheric scenario of self-organization described in [31] had much in 
common with that observed in simulations of the helical-vortex Rayleigh-Bénard convection [26] [27] (see, subsection 2.4.). This did become evident when both scenarios were brought together and discussed in seminars of Montgomery Research Group at Colorado State University in Fort Collins. At this juncture, in February 2006, collaborative Russian-American studies were commenced in order to apply the hypothesis on the turbulent vortex dynamo to examination of tropical cyclogenesis. An initial idea of this paper's author, with which she came to the USA and presented it in those seminars, concerned the implementation of parameterization of helical turbulence [26] in atmospheric modeling systems. Instead, a much more daring and innovative proposal was made by the American side, namely, to try and apply the experience gained at working on [26] [27] for the analysis of the then-newest data [31] obtained by direct numerical near-cloud-resolving modeling of tropical cyclogenesis.

The results of our collaborative research efforts are presented in publications [42]-[48] and in a number of conference abstracts, proceedings, and presentations, most of which are available on-line, e.g., in [49].

\section{Numerical Study of Helical Tropical Cyclogenesis}

Up to the time, when our joint research with American colleagues started, no one had tried to investigate the helical features of atmospheric turbulence by direct (i.e. without introducing any parameterization) numerical simulation resolving the cloud scales. As far as we were then aware, there were close enough to this subject only papers [50] [51] [52], in which calculations of helicity for mature hurricanes were carried out by regional atmospheric models whose space resolution did not allow resolving the most energetic scales of cumulus cloudiness. The fundamentals of turbulence as well as our experience in the helical-vortex Rayleigh-Bénard convection, both discussed above, helped elaborate the statement of the problem and set directing the search by supplying with an important knowledge that was taken into account in our studies.

The basic premise for our studies was that the inverse energy cascade and generation of large-scale structures are possible in helical turbulence characterized by the broken mirror symmetry. Such departure from the mirror symmetry in turbulence can be quantified by helicity of the velocity field. It was fairly obvious to expect helicity generation in the atmospheric turbulence under the influence of the Coriolis force. In this case, it seemed very likely to find in the atmosphere non-zero helicity fluctuations by following expectations of authors [28] [29]. Meanwhile, the non-zero mean helicity needed to provide the vortex dynamo mechanism (see, review [13]) and found in simulations of the so-called laboratory convection [26] [27] was questionable to exist in natural atmospheric conditions.

The foregoing determined the choice of the first step in our studies. We began by calculating the helicity of the velocity field. In papers [42] [43], the first inves-

tigation of tropical cyclone genesis and intensification was conducted from the 
perspective of helical features of atmospheric flows of different scales, which contributed to the organization of the cyclone.

\subsection{Post-Processing of Atmospheric Simulation Data}

To analyze the process of self-organization of moist atmospheric convection observed under conditions of tropical cyclogenesis as posed in [31], a set of helical characteristics was computed, as well as some other integral characteristics of the velocity field which were applied in [27].

The velocity fields used for post-processing in our studies [42]-[48] originated from [31] (in which a more detailed information can be found) and were obtained by use of three-dimensional non-hydrostatic Regional Atmospheric Modeling System (RAMS) comprising time-dependent equations for all three components of velocity, pressure, potential temperature, total water mixing ratio, and cloud microphysics and utilizing an interactive multiple nested grid scheme. For all numerical experiments [31] three nested grids were used. Our post-processing of the model data was carried out on the finest computational grid for subsequent times with a time increment of 10 minutes during 72 hours of numerical experiment. Characteristics were calculated in the computational domain of $276 \times 276 \times 20 \mathrm{~km}$ in Cartesian coordinates by use of uniform finite-difference grid. Throughout the post analysis the vertical increment was equal to $500 \mathrm{~m}$; the horizontal increments were 2 and $3 \mathrm{~km}$. We also applied an analysis of system-scale dynamics from a traditional vortex-centric perspective when the Cartesian model data were transformed into a local cylindrical coordinate system. For these purposes we used the "Diagnostic Package" developed and described in [31] (APPENDIX B).

It is important to point out that no external assumptions were imposed on the fluid motions investigated and described in [42]-[48], i.e., no external forcing terms were imposed to mimic a "helical alpha effect". In other words, the presented results are the outcome of a direct numerical simulation subject to the usual caveats of a sub-grid scale closure that is used to remove small scale motions at the horizontal grid scales of the model $(\sim 3 \mathrm{~km})$.

In [31], nineteen sensitivity experiments were conducted to explore and substantiate the new paradigm of tropical cyclogenesis based on self-organization of vortical moist convection. They were grouped into five categories to examine effects of: (A) horizontal resolution; (B) convective and thermodynamic processes; (C) perturbations in initial MCV structure; (D) absence of latent/sensible heat fluxes or momentum fluxes at ocean surface; and (E) absence of Coriolis parameter.

For our thorough analysis in [42]-[48], six numerical experiments from different categories [31] were chosen-A1, A2, B3, C1, C3, and E1, the last five of which were calculated with the same horizontal grid increment equal to $3 \mathrm{~km}$, while in A1, the increment of $2 \mathrm{~km}$ was applied. In experiments A1, A2, B3, C3, and $\mathrm{E} 1$, a transformation of an initial mid-tropospheric vortex disturbance into a 
surface-concentrated tropical depression vortex was observed. Of these, experiments $\mathrm{A} 1$ and $\mathrm{A} 2$ demonstrated during 72 hours of the computational time the whole evolution of the cyclone from a tropical depression (TD) through a tropical storm (TS) and up to a hurricane (H). Experiments B3, C3, and E1, during the same time, did not reveal any further intensification of the surface-concentrated TD vortices. In case C1, without an initial vortex disturbance, no surface development whatsoever was observed.

\subsection{Helicity Generation on Cloud- and Mesoscales}

In this paper, let us analyze and discuss a few helical characteristics, which have been applied in [42]-[48] and are easily obtained from Formula (1): the three-dimensional helicity density, i.e., helicity values calculated in each point of the finite-difference grid $-h$ and its vertical spatial contribution $h_{z}$, and mean helicity of the forming vortex system introduced as the value of helicity integrated over the whole computational domain and normalized by the number of grid points $-\langle H\rangle$, as well as its integrated spatial contributions $\left\langle H_{\text {hor }}\right\rangle$ and $\left\langle H_{v e r}\right\rangle$

$$
\begin{array}{r}
h=\boldsymbol{v} \cdot \boldsymbol{\omega}=u\left(\frac{\partial w}{\partial y}-\frac{\partial v}{\partial z}\right)+v\left(\frac{\partial u}{\partial z}-\frac{\partial w}{\partial x}\right)+w\left(\frac{\partial v}{\partial x}-\frac{\partial u}{\partial y}\right), \\
\langle H\rangle, \quad\left\langle H_{\text {hor }}\right\rangle, \quad\left\langle H_{v e r}\right\rangle .
\end{array}
$$

It is important to note that the non-zero mean helicity could be generated even in the absence of vertical flows $(w=0)$. However, such is only possible when the horizontal wind is changing with height, i.e., in the occurrence of vertical shear of the horizontal wind. Thus, the non-zero horizontal helicity can be considered as a sign of existing or emerging shear flow. The non-zero vertical helicity, being a product of vertical velocity and vorticity, is an indicator of the presence of vortical convection in the examined area of tropical cyclone formation and allows perfectly the identification of such flows. As our studies [44]-[48] show, the values of vertical helicity are approximately two orders of magnitude less than the values of the horizontal one.

In Figure 3, the helicity density distribution observed in experiment A2 is presented at three horizontal levels, $z=1,4$, and $7 \mathrm{~km}$, along the atmospheric layer height. Three specific moments of time are chosen, that approximately correspond to: (a) $t=12 \mathrm{~h}$-the formation of the secondary circulation; (b) $t=$ $15 \mathrm{~h}$-the tropical depression formation; (c) $t=62 \mathrm{~h}$-when the developing cyclone reaches the hurricane intensity of Category 2 with sustained winds exceeding $42 \mathrm{~m} / \mathrm{s}$.

The color bar indicates the magnitude of $h$ multiplied by $10^{-2}$. Orange, red and dark red regions correspond to strong positive helicity. As part of our examination of the evolution of the three-dimensional helicity field (6), the vertical velocity and vertical vorticity were analyzed also (not shown). This allowed both an identification of the formation of rotating convective structures and determination of their rotational signature, i.e., cyclonic or anticyclonic. 


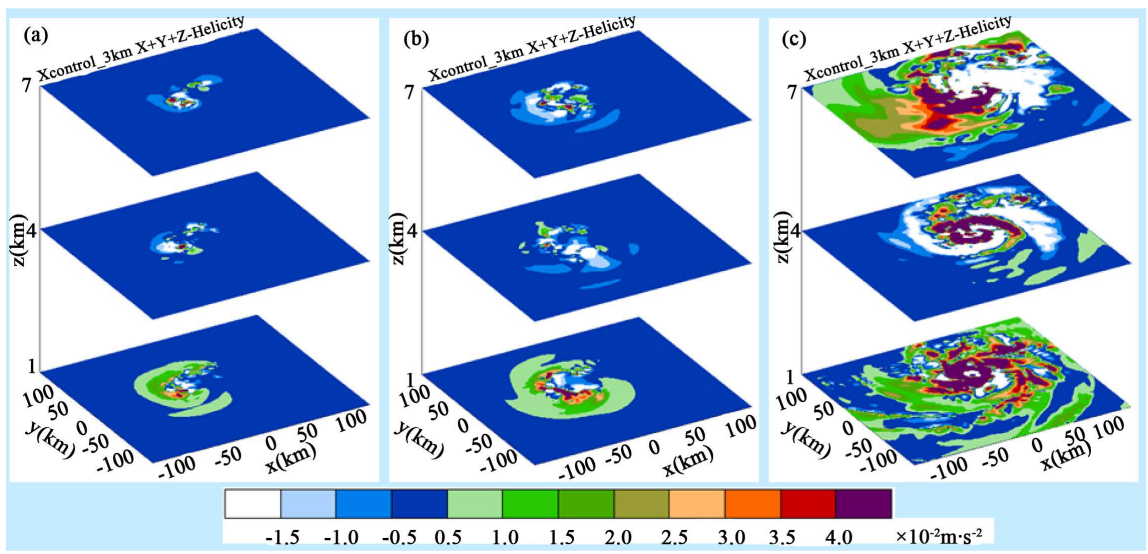

Figure 3 . Helicity density $\left(\times 10^{-2} \mathrm{~m} / \mathrm{s}^{2}\right)$ in three horizontal cross-sections of $276 \times 276 \mathrm{~km}$ at $Z=1 ; 4 ; 7 \mathrm{~km}$ : (a) $t=12 \mathrm{~h}$, (b) $t=15 \mathrm{~h}$, and (c) $t=62 \mathrm{~h}$.

Such rotating vertical flows observed in the field of vertical helicity $h_{z}$ during experiment A2 were first shown in our presentation at the 31st AMS Conference on Hurricanes and Tropical Meteorology [48]. The recorded presentation (mp4) and handout (pdf) are also available on-line [48], in which the color bar (Slide 16) is similar to that in Figure 3, but for $h_{z}$ multiplied by $10^{-4}$.

The undertaken studies [42]-[48] showed the existence of a spectrum of cyclonically rotating deep convection-VHTs of different horizontal and vertical sizes and intensity. VHTs were regarded as vortical coherent structures that spanned more than half of the depth of the tropical troposphere. In contrast with [36], we consider the broader spectrum of such convective structures rather than emphasizing only the most intense updrafts. Some of these helical structures were extremely strong and extended through the bulk of the troposphere. Their ascending vertical velocities sometimes exceeded $30 \mathrm{~m} / \mathrm{s}$.

A successive development of rotating convection was traced and discussed in [31], in which it was demonstrated how a mesoscale tropical depression vortex could develop from cumulonimbus convection as a result of system-scale convergence and upscale vorticity growth. The corresponding helicity evolution was presented in [42]-[44].

A special attention was paid to a process of merging of convective cells interpreted as a manifestation of upscale organization of atmospheric rotating moist convection. As it was shown in [31] [42]-[47], the process of merging was accompanied by not only an emergence of larger and stronger convective structures but also an increase in the background vorticity and helicity in adjacent areas.

In [42]-[48], the integral helicity of a developing mesoscale vortex was also calculated and analyzed. The main and striking result worth to emphasize is that the integrated mean helicity $\langle H\rangle$ was found to be non-zero and, after the first few hours in the vortex evolution, only positive and persistently increasing with time.

Analysis carried out for experiments A2, B3, C3, and E1 from [31] distinctly 
showed that in all four cases there existed an initial period, which was needed for development of intense cloud-scale helical convection and starting of the process of merging of convective structures. As it was pointed out in [44]-[48], the vertical contribution of helicity $\left\langle H_{v e r}\right\rangle$ was particularly instrumental for tracing such changes because it immediately showed an emergence of rotating convection, while a small horizontal contribution $\left\langle H_{\text {hor }}\right\rangle$ generated by vertical shear within the initial MCV was already present since the very beginning. At this stage, duration of which depended on initial conditions in each experiment, the largest horizontal scale of observed convective structures was about $10 \mathrm{~km}$, helicity $\langle H\rangle$ values due to the initial conditions did not exceed $0.2-0.35 \times 10^{12}$ $\mathrm{m}^{4} / \mathrm{s}^{2}$.

During next few hours, an upscale organization continued and led to formation of tropical depression vortices, which had essentially larger scales, with tens kilometers in diameter. The formation of tropical depressions occurred at $t=15$ h (A2-Figure 3(b)); 45 h (B3); 26 h (C3); 27 h (E1). The characteristic values of maximum azimuthal mean tangential velocity within these tropical depressions at $z=1 \mathrm{~km}$ were within $7-9 \mathrm{~m} / \mathrm{s}$, the helicity values increased up to $0.25-0.55 \times$ $10^{12} \mathrm{~m}^{4} / \mathrm{s}^{2}$. Of these vortices, a further intensification of tropical depression was observed only in experiment A2. The process of intensification up to hurricane power was accompanied by a strong increase in helicity values. Thus, at $t=62 \mathrm{~h}$ (Figure $3(\mathrm{c})$ ), the tangential wind and helicity were $42.5 \mathrm{~m} / \mathrm{s}$ and $9.7 \times 10^{12}$ $\mathrm{m}^{4} / \mathrm{s}^{2}$, respectively.

To be sure of the reliability of these new numerical results on the helicity values, an important opportunity was found that allowed their comparison with unique helicity data for intense vortical convection in Hurricane Bonnie (1998) calculated in paper [39] using direct measurements in the atmosphere. Thus, in [39] (Table A1), one can find a few results for helicity integrated over the 0 - 6 $\mathrm{km}$ layer in height that were obtained for a cell motion of zero (what is similar to conditions in our numerical experiments). The highest value, equal to 2578 $\mathrm{m}^{2} / \mathrm{s}^{2}$, was found in Hurricane Bonnie (1998) on August 24 when the maximum surface wind was about $55 \mathrm{~m} / \mathrm{s}$. We calculated the corresponding helicity for experiment A2 [44] [47]. In numerical modeling, results showed to have very high helicity magnitudes between $2000-2400 \mathrm{~m}^{2} / \mathrm{s}^{2}$ during a time span $56-65$ hours when the maximum surface wind was between $33.5 \mathrm{~m} / \mathrm{s}$ and $42.5 \mathrm{~m} / \mathrm{s}$. Within this time interval, the helicity reached its highest local value equal to $2700 \mathrm{~m}^{2} / \mathrm{s}^{2}$, which is close to that found in [39]. Consequently, helicity magnitudes deduced from idealized numerical simulations turned out to be reasonably close to those ones found for intense vortical deep convection in real hurricane conditions.

The non-zero mean helicity within a mesoscale area of tropical cyclone formation, starting from the early hours in the vortex evolution and persistently increasing with time, signifies a break of the mirror symmetry of atmospheric turbulence. The broken mirror symmetry in turbulence is a precondition for the emergence of a large-scale alpha-like instability [5]. This naturally led to the is- 
sue of crucial importance: whether the large-scale helical-vortex instability, known also in fluid mechanics as the turbulent vortex dynamo or hydrodynamic alpha-effect [13], might contribute to the formation of tropical cyclones, and what role might it play.

Meanwhile, the non-zero helicity does not necessarily imply that the large-scale vortex instability is underway. In fact, this only means that the existing departure of the mirror symmetry in turbulence produces an environment conducive to the onset of large-scale instability.

That was a critical point in our research, happened soon after our first results were published [42]. As an obvious continuation aimed at answering the questions, it seemed indispensable to carry out studies of atmospheric turbulence with a focus on analysis of the transport of kinetic energy and helicity over the spectrum of scales. The discovery of an inverse energy cascade during tropical cyclogenesis would become a serious argument in favor of the hypothesis on turbulent vortex dynamo. However, unlike a great amount of investigations, theoretical and numerical, connected with studying the properties of helical turbulence in the general and magnetohydrodynamics (see, for example, [53] [54], and references therein), at that time, there were no any similar studies on atmospheric turbulence in conditions corresponding to tropical cyclogenesis. They were absent even for the classical case of non-helical turbulence, which could be useful to us in order to correctly take into account all the specific features inherent in the conditions of the formation of tropical cyclones. As our further experience has shown, such features do exist.

Having postponed for a while the problem of turbulent statistics, the work on which required the search and involvement of a highly qualified expert in both turbulence and tropical cyclone fields, it was decided to begin with a search for the large-scale helical-vortex instability.

To this end, a numerical approach [26] [27], based on a joint analysis of helical and energetic characteristics of the velocity field, was adapted for the use of atmospheric data [31]. The approach was applied to examine peculiarities of the evolution of helicity and kinetic energy during upscale vorticity organization observed in near-cloud-resolving simulations [31].

\section{Role of Vortical Hot Towers in Providing the "Vortex-Motive" Force}

In [31], a dipolar structure of the vorticity anomalies was found that was approximately collocated with the hot towers. This reminds a similar finding described for midlatitude supercells in [55] [56] [57], and in many other publications that followed them. The works presented a mechanism to produce a vortex dipole by the updraft tilting the environmental horizontal vorticity into the vertical one. At the same time, it was substantiated in [57], how this process could generate helicity and was shown that rotating supercell thunderstorms had to possess the high helicity. 
Figure 4 is presented to illustrate how the vertical vorticity and helicity were generated during the formation of a VHT in numerical experiments [31]. For this purpose, Figure 9(b) showing the VHT, and Figure 10 on vortex tilting were borrowed from [31] and combined.

In the majority of numerical experiments in [31], the simulation was initialized with a weak midlevel vortex elevated above the sea surface, with a maximal mean tangential wind at $z=4 \mathrm{~km}$. The vortex had a basic-state cyclonic tangential velocity field that increased in magnitude with height below $z=4 \mathrm{~km}$ and decreasing above. This provided an environment rich in horizontal and vertical vorticity. Ignoring buoyant effects, we could consider the horizontal vorticity profile at the initial time as being due solely to the vertical shear of horizontal wind of the initial mesoscale convective vortex (MCV). Such vertical shear profile will generate a radial vorticity profile that, when tilted upward by an updraft, generates negative (positive) relative vertical vorticity anomalies on the radially inward side of the updraft below (above) $z=4 \mathrm{~km}$. Evolving convection tilts ambient horizontal vorticity into the vertical while at the same time stretching MCV-generated vertical vorticity. As some updraft intensifies to become a hot tower, both ambient and tilting generated vertical vorticity is stretched even more, leading to a strong convectively generated vertical vorticity anomaly.

The described mechanism was interpreted in [44] [45] [46] [47] as an effective way for helicity generation. In work [44], a comprehensive quantitative analysis was carried out for this process by using the data of six numerical experiments of [31]. It was found that maximal values of helicity $\langle H\rangle$ generated by the interaction between the very first updraft complex and MCV within the initial two

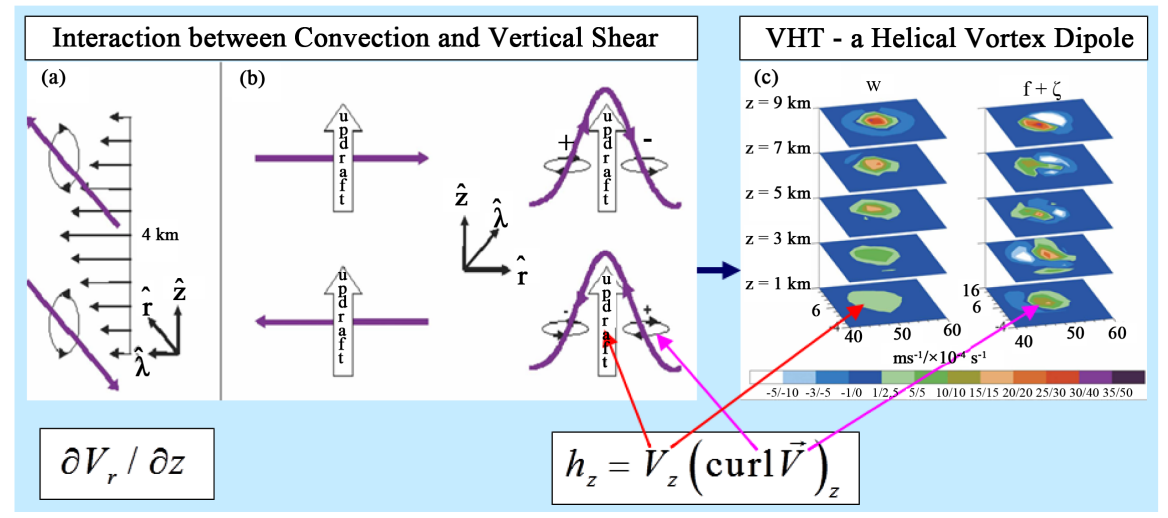

Figure 4. Formation of a VHT. (a) \& (b) Schematic of vortex tilting within the initial MCV [31]. Purple lines represent vortex filaments. (a) Radial vorticity generated by vertical shear profile of initial MCV; (b) Updraft tilts radial vortex filament upward, generating a vertical vorticity dipole with negative relative vorticity radially inward (outward) at heights below (above) $z=4 \mathrm{~km}$; (c) Vertical velocity $w(\mathrm{~m} / \mathrm{s}$ ) and absolute vertical vorticity $\eta\left(\times 10^{-4} 1 / \mathrm{s}\right)$ signatures associated with deep cumulus convection in experiment $\mathrm{A} 1$ at $t=40 \mathrm{~min}$. Horizontal cross sections are $20 \mathrm{~km} \times 20 \mathrm{~km}$ subdomains centered at $x=50$ $\mathrm{km}, y=6 \mathrm{~km}$. The existence of a strong vorticity dipole collocated with the core of the hot tower and its orientation suggest tilting of ambient vorticity associated with the initial MCV. 
hours of those experiments were between $0.2-0.35 \times 10^{12} \mathrm{~m}^{4} / \mathrm{s}^{2}$. Let us note, that the first updraft was initiated by a local heating at low levels, $z=2 \mathrm{~km}$ [31]. The heating was applied for $300 \mathrm{~s}$ in order to stimulate cumulus convection in the local environment of the MCV. In [44] [58], thanks to this, it was possible to quantitatively examine how helicity could be generated by a single updraft, and evaluate the effect of initial conditions on this process.

At later times in the simulations the convergence/stretching of near surface $(0$ $<z<2 \mathrm{~km}$ ) vorticity by the convective plumes dominated the generation of vorticity by tilting processes [31]. Meanwhile, as our studies [44] [45] [46] [47] [48] showed, the latter play a crucial role in providing the linkage of a forming transverse circulation with the existing tangential one, and in maintaining such linkage during the whole evolution of a tropical cyclone. Helicity is just a quantitative measure of this linkage.

Moreover, Figure 4 can serve perfectly for giving an interpretation for the hypothetical "vortex-motive" force described by Formula (4), and consequently, the realization of the vortex dynamo in the atmosphere. The component representation of the force in Formula (5) shows the interaction between vertical wind shear (the first and second term) and vortical convection (the third term) while coefficient $C$ characterizes an energy supply due to sensible and latent heat release (an appropriate discussion and its quantitative estimate for pre-hurricane conditions are given in [26]). Thus, the vortical moist convection, discovered in 2004, becomes the crucial link that, before this finding, was only hypothetical in mathematical model (3) and which can give a real life to the vortex dynamo theory and promote its application for important diagnostic purposes.

Further, we briefly demonstrate, following [44] [45] [46] [47] [48], how the concept of vortex dynamo can be applied for diagnosing the commencement time of tropical cyclogenesis.

\section{Diagnosis of Tropical Cyclogenesis}

The results of studies [13] [26] [27] suggested what first step might be tried. They showed that the first sign of the hypothesized large-scale helical-vortex instability should be generation of the linkage of tangential (primary) and transverse (secondary) circulation on the system scale, and resulting of it, a positive feedback that makes the forming hurricane vortex energy-self-sustaining. Such feedback may reveal itself in mutual intensification of both circulations. Accordingly, because the helicity is a quantitative measure of the linkage, it was necessary to examine how its production was accomplished both on small and large scales (Section 3.4.). The helicity was found to be essentially non-zero. Then, we should analyze the evolution of kinetic energy divided into two parts, of primary $\left\langle E^{P}\right\rangle$ and secondary $\left\langle E^{S}\right\rangle$ circulation, similarly to that was performed in [26] [27]

$$
\langle E\rangle=\left\langle E^{P}\right\rangle+\left\langle E^{S}\right\rangle
$$


To quantitatively diagnose an emerging feedback loop between the primary and secondary circulation, we examined the kinetic energy of both circulations calculated as squares of corresponding components of velocity in the cylindrical coordinates, integrated over the computational domain and normalized by number of grid points.

The kinetic energy evolution in experiments A2, B3, C3, and E1 is shown in Figure 5. In the following discussion, we will also refer to helicity evolution in Figure 3 as well as a set of other data gained from snapshots of spatial velocity, vorticity, helicity and temperature fields at horizontal and vertical cross-sections (not shown).

To those readers, who are interested in looking deeper into detailed hydroand thermodynamics of tropical cyclone formation, an impressive visualization in [31] can be recommended to more illustrate the current discussion. Study [31] also presents numerous visualizations of azimuthally averaged mean hydroand thermodynamic fields used for diagnosis of main milestones (e.g. the formation of secondary circulation, tropical depression formation) in a tropical cyclone evolution; they are not shown in this paper.

In [44]-[48], we proposed how tropical cyclogenesis may be identified based on the new knowledge regarding helical flow organization and the important role of VHTs in this process.

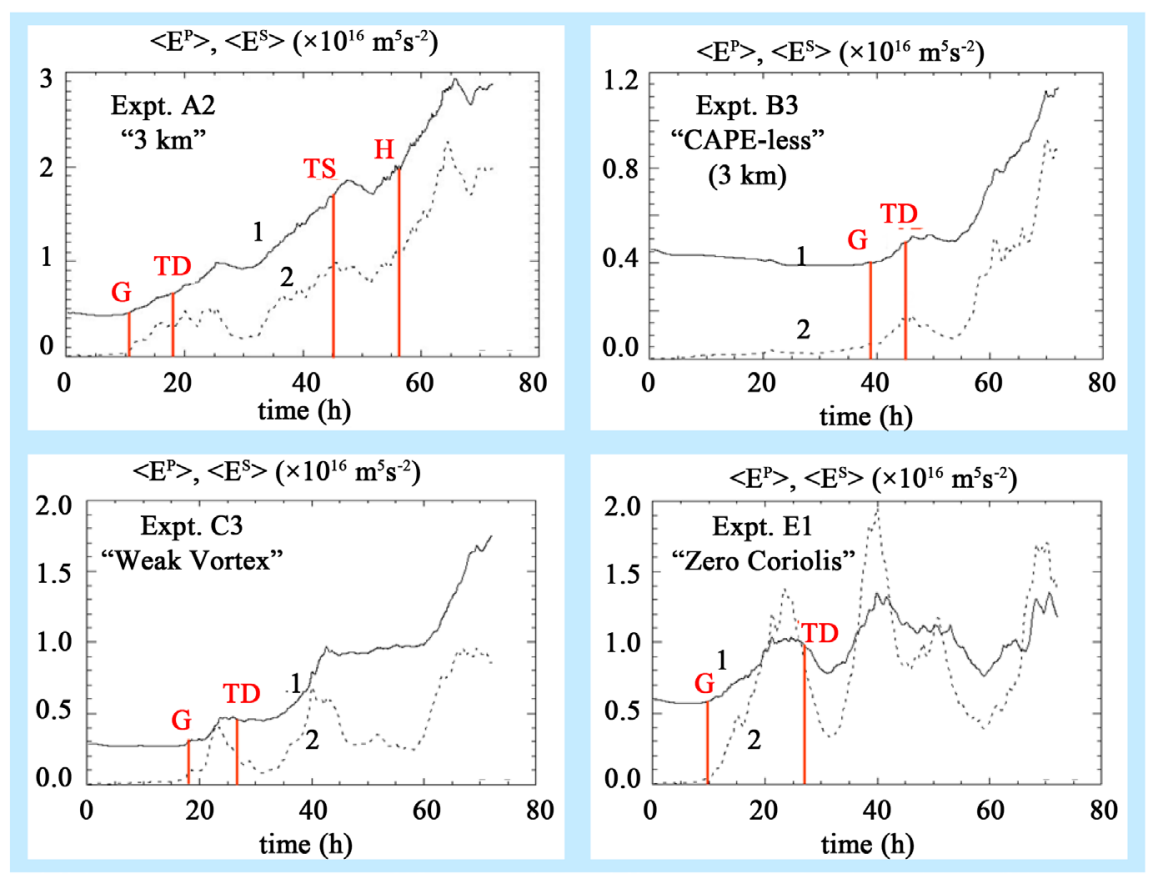

Figure 5. Evolution of the kinetic energy of primary (1) and secondary (2) circulation, shown respectively by solid and dotted line, in four numerical experiments of [31]. Red lines mark the energy magnitudes, $\left\langle E^{P}\right\rangle$ and $\left\langle E^{S}\right\rangle$, at the time moments corresponding to: $\mathrm{G}-\mathrm{a}$ mutual intensification of both circulations starts and the nascent vortex becomes energy-self-sustaining; TD-the tropical depression is formed; TS-the vortex reaches the intensity of tropical storm; $\mathrm{H}$-the vortex intensifies up to hurricane strength. 
In numerical experiments A2, B3, C3, and E1 [31], a weak tangential circulation existed in the low and mid troposphere (with a maximal tangential wind at $4 \mathrm{~km}$ altitude) from the beginning due to the initial mesoscale convective vortex. In these four cases, the kinetic energy of the primary circulation $\left\langle E^{P}\right\rangle$ was between $0.3-0.6 \times 10^{16} \mathrm{~m}^{5} / \mathrm{s}^{2}$ (Figure 5). Initially, the secondary transverse circulation was absent, $\left\langle E^{S}\right\rangle=0$.

During an initial time interval, which lasts about 10 hours in A2 and E1, 15 16 hours in C3, and near 40 hours in B3, the tangential circulation is slightly weakening against its initial energy value (Figure 5), whilst the energy of the secondary circulation slowly increases. Unlike the initially coherent tangential circulation, the radial and vertical flows contributing to the secondary circulation are chaotic and weak enough, that such motions result in a small but nonzero kinetic energy, $\left\langle E^{S}\right\rangle \neq 0$. The first rotating convective flows become visible at near the half of the time interval and a process of merging of convective cells commences. This phenomenon was described in detail, analyzed quantitatively and interpreted in [31] as a manifestation of upscale organization of atmospheric rotating moist convection. The process of merging is aided by the secondary circulation, which is forced by the radial gradient of the aggregate latent heating of the cumulus cloud population. The merging is accompanied by not only an emergence of larger scale and stronger convective structures but also an increase in the background vorticity and helicity in adjacent areas. Convective activity is progressing in all four experiments by producing a population of convective updrafts, which interact with each other by straining and partial or complete merger. During these vortex-cloud interactions some of the vertical vorticity anomalies are enhanced relative to their prior values. At this time, there exists a few cyclonically rotating convective cores-VHTs-of different horizontal and vertical sizes and intensity. The most intense updrafts are approximately $10 \mathrm{~km}$ in diameter and grow gradually with time from $4-6 \mathrm{~km}$ height to $8-10 \mathrm{~km}$ height.

The rotating convective cells start to generate a non-vanishing and increasing third (vertical) contribution of helicity near $t=7-8 \mathrm{~h}$ in A2 and E1, $14-15 \mathrm{~h}$ in C3, and 18 - $20 \mathrm{~h}$ in B3 and, thereby, a local linkage of vortex lines of horizontal and vertical flow components in a vicinity of each rotating updraft. Each rotating convective structure contributes simultaneously to both the tangential and overturning circulation, namely, by its vertical vorticity to the former and by its vertical motion to the latter. Thus, such a structure represents a natural link between the circulations on the local scale whilst the developed population should help link the primary and secondary circulations on the system scale.

A degree of such linkage is measured by helicity.

Thus, in experiment A2, an increase in the vertical contribution of helicity from zero up to approximately $0.5 \times 10^{10} \mathrm{~m}^{4} / \mathrm{s}^{2}$ within $6-9 \mathrm{~h}$ is associated with an amplification of horizontal and total helicity from $0.15 \times 10^{12} \mathrm{~m}^{4} / \mathrm{s}^{2}$ up to 0.3 $\times 10^{12} \mathrm{~m}^{4} / \mathrm{s}^{2}$. Just at this time, $t=6 \mathrm{~h}$, a slight yet distinct increase in the kinetic energy $\left\langle E^{S}\right\rangle$ starts. 
Near $t=10 \mathrm{~h}$ in $\mathrm{A} 2$ and E1, $20 \mathrm{~h}$ in C3, and $40 \mathrm{~h}$ in B3, one can observe dramatic changes in the flow intensity-the kinetic energy of the transverse circulation, $\left\langle E^{S}\right\rangle$, increases sharply and soon after this, kinetic energy of the tangential circulation, $\left\langle E^{P}\right\rangle$, starts to increase as well (Figure 5). Our analysis of the flow structure and dynamics shows that near the time when the mutual intensification of circulations starts, in all experiments, an intense helical updraft-VHT of about 12 - $14 \mathrm{~km}$ in height appears (Figure 3(a)). The VHT is found to be strong enough to generate a large transverse circulation, of tens of kilometers in horizontal directions and throughout the whole troposphere layer up to $14 \mathrm{~km}$ in height. The circulation is characterized by a radial inflow near the surface and in the middle troposphere, rising flow in the center and radial outflow in the upper levels. These flows in the four experiments are characterized by the following values for azimuthally-averaged mean velocity: $1.30-2.05 \mathrm{~m} / \mathrm{s}$-inflow, $0.55-1.0 \mathrm{~m} / \mathrm{s}$-rising flow, $0.85-1.60 \mathrm{~m} / \mathrm{s}$-outflow. The overall intensity of vortical convection at this moment can be evaluated by the vertical contribution of helicity, $\left\langle H_{v e r}\right\rangle$ which varies between $0.1-0.6 \times 10^{10} \mathrm{~m}^{4} / \mathrm{s}^{2}$. The helicity, $\langle H\rangle$, is about $0.08-0.23 \times 10^{12} \mathrm{~m}^{4} / \mathrm{s}^{2}$. This gives a start to the formation of a stable system-scale (hundreds of kilometers horizontally) secondary circulation during the next $1-2 \mathrm{~h}$. The secondary circulation is sustained and linked with the primary circulation by the strong VHT and a number of smaller and less intense rotating convective cores (Figure $3(\mathrm{a})$ ).

Once the linkage on the system scale is formed, this marks a critical point in a process of tropical cyclone formation when the vortex becomes self-sustaining. The time after which both $\left\langle E^{S}\right\rangle$ and $\left\langle E^{P}\right\rangle$ mutually increase may be considered a practical definition for the moment of tropical cyclogenesis- " $G$ ". The simulations indicate that a positive feedback formed between the two circulations is sustained by convective instability and vortical convection in the central region of the developing circulation. The convective instability there is maintained primarily by latent heat fluxes from the underlying sea surface, which need not increase with wind speed [59]. The existence of such rising warm flows suggests a release of potential energy that is converted into kinetic energy of developing large-scale helical vortex. The active feedback provides energy exchange between the primary and secondary circulation and further mutual intensification. During subsequent development in experiment A2, a hurricane $(\mathrm{H})$ vortex was formed (Figure 3(c) and Figure 5) while B3, C3, and E1 resulted in formation of tropical depression (TD) vortices. No intensification of the formed TDs within 72 hours of simulation time was observed. In this connection it is important to point out that experiment A2 is characterized by the highest value of the vertical helicity, which far exceeds similar characteristics in other experiments [44] [46]. The vertical helicity is a quantitative measure of the intensity of vortical convection. It may be considered as a measure of VHTs effectiveness in maintaining the process of horizontal vorticity transformation into the vertical one, amplification of the latter, and generation of the linkage be- 
tween the system-scale tangential and transverse circulations.

\section{Conclusions}

We have provided an overview of the efforts undertaken to apply the fundamental ideas on self-organization in helical turbulence to study the formation of tropical cyclones in the atmosphere, and also contributed to this problem by a new suggestion concerning the analogy between the vortex dynamo in the atmosphere and MHD dynamo in electrically conducting medium. Beginning with the short excursus to the 1980s, when the hypothesis on the turbulent vortex dynamo was put forward and wide-ranging research program was arranged to test it, we have emphasized recent results of our collaborative Russian-American investigations.

1) Our first finding [42] concerned the non-zero and persistently increasing with time mean helicity of atmospheric turbulence within an area of a developing tropical cyclone. This has become the first example of such phenomenon in a natural system, namely, the tropical atmosphere of the Earth. This meant a violation of the mirror symmetry of turbulence, which, according to the theory of turbulence, is conducive to the emergence of large-scale vortex instability.

2) By adapting for post-processing of atmospheric simulation data our earlier developed approach [26] [27], which was tested in the search for a large-scale vortex instability in the helical-vortex Rayleigh-Bénard convection, we have discovered similar instability in conditions of tropical cyclogenesis [44] [45] [46] [47] [48]. The onset of instability was defined as the start of mutual intensification of the primary and secondary circulation making a nascent hurricane vortex energy-self-sustaining. This may be considered as a commencement of tropical cyclogenesis shown as "G" in Figure 5. It must be stressed that the observed new instability begins several hours earlier than the vortex of tropical depression is formed ("TD" in Figure 5). At present, it is the latter that is usually declared in meteorological observations as the formation of a tropical cyclone. If the discovered instability is reliably confirmed for tropical cyclones observed in real natural conditions, it may be suggested to consider the emergence of this instability as the commencement of the Genesis stage, and the formation of Tropical Depression as the completion of this stage in evolution of developing intense vortex. This may help develop a universally accepted definition of tropical cyclogenesis that currently does not exist.

3) For the first time in tropical cyclone research, we have emphasized a role of special topology of forming vortex provided by interaction of motions of different scales [44] [45] [46] [47] [48]. It has been found that the newly forming mesoscale vortex becomes energy-self-sustaining when a helical structure of the system-scale circulation organizes. Such helical mesoscale organization is only possible due to the linkage of primary tangential and secondary transverse circulation, which is realized through rotating convective structures of cloud scales-VHTs. 
For purposes of quantitative diagnosis, we have analyzed the evolution of energetics and structure of the forming vortex. The integral kinetic energy of primary and secondary circulation was applied to diagnose the onset of large-scale vortex instability (Figure 5). A pseudoscalar-helicity of the velocity field (helicity density, integral helicity as well as its horizontal and vertical contribution) was applied to quantitatively analyze the topology.

4) The process of helicity generation in conditions of tropical cyclogenesis was examined in [42]-[48], and with a special focus on how this is realized by a single updraft generated by a local heating at low levels in [44] [58]. A detailed discussion is given in the present paper. We can summarize that the VHTs appear as a result of interaction between cloud moist convection and vertical wind shear. Each convective updraft generates the vertical vorticity by tilting of horizontal vortex filaments and amplifies it by stretching. This process provides a linkage of horizontal and vertical vortex lines and results in helicity generation on a local cloud scale whilst an evolving population of VHTs of different sizes and intensity during tropical cyclone evolution ensures the linkage of circulations on mesoscales. Helicity dynamics allows tracing such upscale flow organization.

5) Thus, due to the crucial role of topology in the new instability and role of VHTs in providing this, the helicity can be suggested as a measure to quantify the chaotic influence resulting from moist convection.

6) In the present paper, we discuss and substantiate the key role of VHTs as a necessary element to provide the dynamo effect. Based on the mathematical model for the turbulent vortex dynamo, an analogy is traced between the role of interaction "moist convection-vertical wind shear" in creating the vortex dynamo in the atmosphere and the role of the mean electromotive force providing the MHD dynamo in electrically conducting medium.

Although some successful steps have been undertaken to substantiate the contribution of the turbulent vortex dynamo to tropical cyclogenesis, further research is needed:

- It is indispensable to study the turbulence statistics with a focus on analysis of the transport of kinetic energy and helicity over the spectrum of scales. The discovery of an inverse energy cascade would become a serious argument in order to confirm the vortex dynamo.

- Another challenging task is connected with the search for a possible threshold of discovered instability. Bearing in mind the above analysis based on the vortex dynamo model and discussion for the vortex-motive force, it seems useful to combine helicity with Convective Available Potential Energy (CAPE), see, e.g. [39] [40]. Similarly to approaches well known for midlatitude severe storms and tornadoes, this may help introduce a criterion for the onset of large-scale helical-vortex instability during tropical cyclone formation.

- The discovery of the instability threshold would allow us to recall the idea on 
impacting on tropical cyclogenesis, which was investigated within the framework of the Soviet Program, in order to consider it using the most advanced tools of modern science.

- To implement the above suggestions, it seems very promising to use as a basis for investigations a platform similar to the "Hurricane Nature Run" developed and applied in [60]. One could propose to develop an analogous "Genesis Nature Run" for the Caribbean Summer 2010 when the trio of campaigns-GRIP, IFEX, and PREDICT were underway and resulted in collection of great amount of diverse data for observed tropical cyclones.

Meanwhile, it would be possible to begin without postponing and to apply our approach presented in Section 6 to a real case of observed tropical cyclone. This implies a combination of our approach with the theory of tropical cyclogenesis in an easterly wave critical layer [38], which allows with high accuracy to predict the location of the potential tropical cyclogenesis and is adapted for use in global numerical models [61], and successfully applied in practice [34]. Such combination allows locating an area, where the highest space resolution should be applied for diagnosis of large-scale helical-vortex instability. A couple of very intriguing candidates of tropical cyclones can be proposed-Hurricane Harvey (2017) and Hurricane Karl (2010). As to the genesis of the latter, the author was very lucky to be a witness during a research flight.

\section{Acknowledgements}

This work was supported in part by the Russian Foundation for Basic Research (RFBR) under Grant 16-05-00551a. The results based on near-cloud-resolving numerical simulation could only be obtained in close collaboration with my American co-author in many works, M.T. Montgomery, and his colleagues M.E. Nicholls, and S. Barve. I am deeply grateful for discussions and recommendation about writing the review to R.Z. Sagdeev, the co-author of the vortex dynamo theory and Director of Space Research Institute in the 1970-80s, who supervised the Soviet Research Program discussed in the Introduction. I also thank the referees for their valuable comments, which have led to significant improvements in presentation.

\section{References}

[1] Moiseev, S.S., Sagdeev, R.Z., Tur, A.V., Khomenko, G.A. and Yanovsky, V.V. (1983) Theory of the Origin of Large-Scale Structures in Hydrodynamic Turbulence. Soviet Physics-JETP, 58, 1149-1157.

[2] Moiseev, S.S., Sagdeev, R.Z., Tur, A.V., Khomenko, G.A. and Shukurov, A.M. (1983) Physical Mechanism of Amplification of Vortex Disturbances in the Atmosphere. Soviet Physics, Doklady, 28, 925-928.

[3] Moffatt, H.-K. (1969) The Degree of Knottedness of Tangled Vortex Lines. Journal of Fluid Mechanics, 35, 117-129. https://doi.org/10.1017/S0022112069000991

[4] Moffatt, H.-K. and Tsinober, A. (1992) Helicity in Laminar and Turbulent Flow. Annual Review of Fluid Mechanics, 24, 281-312. 
https://doi.org/10.1146/annurev.fl.24.010192.001433

[5] Frisch, U. (1995) Turbulence: The Legacy of A.N. Kolmogorov. Cambridge University Press, Cambridge, UK.

[6] Moffatt, H.-K. (2014) Helicity and Singular Structures in Fluid Dynamics. Proceedings of the National Academy of Sciences of the United States of America, 111, 3663-3670. https://doi.org/10.1073/pnas.1400277111

[7] Moffatt, H.-K. (1978) Magnetic Field Generation in Electrically Conducting Fluids. Cambridge University Press, Cambridge, London, New York, Melbourne.

[8] Steenbeck, M., Krause, F. and Rädler, K.-H. (1966) A Calculation of the Mean Electromotive Force in an Electrically Conducting Fluid in Turbulent Motion, under the Influence of Coriolis Forces. Zeitschrift für Naturforschung, 21A, 369-376. English Translation: Roberts \& Stix (1971), 29-47. (From German)

[9] Krause, F. and Rädler, K.-H. (1980) Mean-Field Magnetohydrodynamics and Dynamo Theory. Academie-Verlag, Berlin.

[10] Frisch, U., She, Z.S. and Sulem, P.L. (1987) Large-Scale Flow Driven by the Anisotropic Kinetic Alpha Effect. Physica D: Nonlinear Phenomena, 28, 382-392. https://doi.org/10.1016/0167-2789(87)90026-1

[11] Fortov, V.E., Ivanov, M.F., Ivlev, A.V., Gnedin, Y.N. and Klumov, B.A. (1996) Collision of Comet Shoemaker-Levy 9 with Jupiter: What Did We See. Physics-Uspekhi, 39, 363-392. https://doi.org/10.1070/PU1996v039n04ABEH000142

[12] Ivanov, M.F., Galburt, V.A. and Fortov, V.E. (1996) On Possible Mechanism of Large-Scale Disturbances Formation in Jovian Atmosphere Initiated by a Fall of Comet Shoemaker-Levy 9 Fragments. Journal of Experimental and Theoretical Physics Letters, 63, 813-817. https://doi.org/10.1134/1.567096

[13] Levina, G.V., Moiseev, S.S. and Rutkevich, P.B. (2000) Hydrodynamic Alpha-Effect in a Convective System. Advances in Fluid Mechanics, 25, 111-162.

[14] Levina, G.V., Startsev, S.E., Zimin, V.D. and Moiseev, S.S. (1996) About Another Physical Mechanism of Large-Scale Structures Generation at Turbulent Convection in the Horizontal Fluid Layer. Physics and Chemistry of the Earth Parts, 21, 563-565. https://doi.org/10.1016/S0079-1946(97)81158-5

[15] Zimin, V.D., Levina, G.V., Veiber, E.E., Veselov, V.M., Gerbek, E.E., et al. (1991) Experimental Studies of Large-Scale Structures Origination in Tropical Atmosphere (Expedition “Typhoon-89”). Proceedings of International Symposium on Generation of Large-Scale Structures in Continuous Media, Nonlinear Instability, Chaos and Turbulence, Singapore, 327-336.

[16] Moiseev, S.S., Rutkevich, P.B., Tur, A.V. and Yanovskii, V.V. (1988) Vortex Dynamo in a Convective Medium with Helical Turbulence. Soviet Physics-JETP, 67, 294-299.

[17] Lupyan, E.A., Mazurov, A.A., Rutkevich, P.B. and Tur, A.V. (1992) Generation of Large-Scale Vortices through the Action of Spiral Turbulence of a Convective Nature. Soviet Physics-JETP, 75, 833-838.

[18] Lupyan, E.A., Mazurov, A.A., Rutkevich, P.B. and Tur, A.V. (1993) Scenario of Large-Scale Vortex Structure Development in the Atmosphere. Doklady AN, 329, 720-722.

[19] Rutkevich, P.B. (1993) Equation for the Rotational Instability Due to Convective Turbulence and the Coriolis Force. Journal of Experimental and Theoretical Physics, 77, 933-938.

[20] Rutkevich, P.B. (1994) Generating Properties of Convective Turbulence in the Cori- 
olis Force Field. Doklady AN, 334, 44-46.

[21] Kopp, M., Tur, A. and Yanovsky, V. (2015) The Large Scale Instability in Rotating Fluid with Small Scale Force. Open Journal of Fluid Dynamics, 5, 128-138. https://doi.org/10.4236/ojfd.2015.52015

[22] Kopp, M.I., Tur, A.V. and Yanovsky, V.V. (2017) Nonlinear Vortex Dynamo in a Rotating Stratified Moist Atmosphere. Journal of Experimental and Theoretical Physics, 124, 1010-1022. https://doi.org/10.1134/S1063776117060127

[23] Hide, R. (1976) A Note on Helicity. Geophysical Fluid Dynamics, 7, 157-161. https://doi.org/10.1080/03091927508242617

[24] Kurgansky, M.V. (1993) Generation of Helicity in a Moist Atmosphere. Izvestiya, Atmospheric and Oceanic Physics, 29, 444-448.

[25] Kurgansky, M.V. (1998) Vorticity Generation in a Moist Atmosphere. Izvestiya, Atmospheric and Oceanic Physics, 34, 156-161.

[26] Levina, G.V. (2006) Parameterization of Helical Turbulence in Numerical Models of Intense Atmospheric Vortices. Doklady Earth Sciences, 411, 1417-1421. https://doi.org/10.1134/S1028334X06090182

[27] Levina, G.V. and Burylov, I.A. (2006) Numerical Simulation of Helical-Vortex Effects in Rayleigh-Bénard Convection. Nonlinear Processes in Geophysics, 13, 205-222. https://doi.org/10.5194/npg-13-205-2006

[28] Levich, E. and Tzvetkov, E. (1984) Helical Cyclogenesis. Physics Letters A, 100, 53-56. https://doi.org/10.1016/0375-9601(84)90354-2

[29] Levich, E. and Tzvetkov, E. (1985) Helical Inverse Cascade in Three-Dimensional Turbulence as a Fundamental Dominant Mechanism in Mesoscale Atmospheric Phenomena. Physics Reports, 128, 1-37. https://doi.org/10.1016/0370-1573(85)90036-5

[30] Hendricks, E.A., Montgomery, M.T. and Davis, C.A. (2004) The Role of "Vortical" Hot Towers in the Formation of Tropical Cyclone Diana (1984). Journal of the Atmospheric Sciences, 61, 1209-1232. https://doi.org/10.1175/1520-0469(2004)061<1209:TROVHT>2.0.CO;2

[31] Montgomery, M.T., Nicholls, M.E, Cram, T.A. and Saunders, A.B. (2006) A Vortical Hot Tower Route to Tropical Cyclogenesis. Journal of the Atmospheric Sciences, 63, 355-386. https://doi.org/10.1175/JAS3604.1

[32] Reasor, P.D., Montgomery, M.T. and Bosart, L.F. (2005) Mesoscale Observations of the Genesis of Hurricane Dolly (1996). Journal of the Atmospheric Sciences, 62, 3151-3171. https://doi.org/10.1175/JAS3540.1

[33] Houze Jr., R.A. (2010) Clouds in Tropical Cyclones. Monthly Weather Review, 138, 293-344. https://doi.org/10.1175/2009MWR2989.1

[34] Montgomery, M.T., Davies, C., Dunkerton, T., Wang, Z., Velden, C., et al. (2012) The Pre-Depression Investigation of Cloud Systems in the Tropics (PREDICT) Experiment: Scientific Basis, New Analysis Tools, and Some First Results. Bulletin of the American Meteorological Society, 93, 153-172. https://doi.org/10.1175/BAMS-D-11-00046.1

[35] Kilroy, G., Smith, R.K. and Montgomery, M.T. (2017) A Unified View of Tropical Cyclogenesis and Intensification. Quarterly Journal of the Royal Meteorological Society, 143, 450-462. https://doi.org/10.1002/qj.2934

[36] Riehl, H. and Malkus, J.S. (1958) On the Heat Balance in the Equatorial Trough Zone. Geophysica, 6, 503-538.

[37] Houze Jr., R.A., Lee, W.C. and Bell, M.M. (2009) Convective Contribution to the 
Genesis of Hurricane Ophelia (2005). Monthly Weather Review, 137, 2778-2800. https://doi.org/10.1175/2009MWR2727.1

[38] Dunkerton, T.J., Montgomery, M.T. and Wang, Z. (2009) Tropical Cyclogenesis in a Tropical Wave Critical Layer: Easterly Waves. Atmospheric Chemistry and Physics, 9, 5587-5646. https://doi.org/10.5194/acp-9-5587-2009

[39] Molinari, J. and Vollaro, D. (2008) Extreme Helicity and Intense Convective Towers in Hurricane Bonnie. Monthly Weather Review, 136, 4355-4372. https://doi.org/10.1175/2008MWR2423.1

[40] Molinari, J. and Vollaro, D. (2010) Distribution of Helicity, CAPE, and Shear in Tropical Cyclones. Journal of the Atmospheric Sciences, 67, 274-284. https://doi.org/10.1175/2009JAS3090.1

[41] Wikipedia, the Free Encyclopedia. https://en.wikipedia.org/wiki/Hot_tower

[42] Levina, G.V. and Montgomery, M.T. (2010) A First Examination of the Helical Nature of Tropical Cyclogenesis. Doklady Earth Sciences, 434, 1285-1289. https://doi.org/10.1134/S1028334X1009031X

[43] Levina, G.V. and Montgomery, M.T. (2011) Helical Scenario of Tropical Cyclone Genesis and Intensification. Journal of Physics. Conference Series, 318, Article ID: 072012. https://doi.org/10.1088/1742-6596/318/7/072012

[44] Levina, G.V. (2013) Helical Organization of Tropical Cyclones, Preprint NI13001-TOD. Isaac Newton Institute for Mathematical Sciences, Cambridge, UK. http://www.newton.ac.uk/science/publications/preprints

[45] Levina, G.V. and Montgomery, M.T. (2014) Numerical Diagnosis of Tropical Cyclogenesis Based on a Hypothesis of Helical Self-Organization of Moist Convective Atmospheric Turbulence. Doklady Earth Sciences, 458, 1143-1148. https://doi.org/10.1134/S1028334X14090189

[46] Levina, G.V. and Montgomery, M.T. (2014) Tropical Cyclogenesis: A Numerical Diagnosis Based on Helical Flow Organization. Journal of Physics. Conference Series, 544, Article ID: 012013. https://doi.org/10.1088/1742-6596/544/1/012013

[47] Levina, G.V. and Montgomery, M.T. (2015) When Will Cyclogenesis Commence Given a Favorable Tropical Environment?: To the $30^{\text {th }}$ Anniversary of the Hypothesis on the Turbulent Vortex Dynamo and Dedicated to the Memory of Soviet-Russian Scientist, Professor Semen Samoilovich Moiseev. Procedia IUTAM, 17, 59-68. https://doi.org/10.1016/j.piutam.2015.06.010

[48] Levina, G.V. and Montgomery, M.T. (2014) Helical Nature of Tropical Cyclogenesis: WHEN Will a Nascent Vortex Become Self-sustaining? Abstracts of the 31 st AMS Conference on Hurricanes and Tropical Meteorology, San Diego, CA, 31 March-4 April 2014.

https://ams.confex.com/ams/31Hurr/webprogram/Paper243281.html

[49] https://www.researchgate.net/profile/Galina_Levina

[50] Xu, Y. and Wu, R. (2003) The Conservation of Helicity in Hurricane Andrew (1992) and the Formation of the Spiral Rainband. Advances in Atmospheric Sciences, 20, 940-950. https://doi.org/10.1007/BF02915517

[51] Han, Y., Wu, R. and Fang, J. (2006) Shearing Wind Helicity and Thermal Wind Helicity. Advances in Atmospheric Sciences, 23, 504-512. https://doi.org/10.1007/s00376-006-0504-5

[52] Glebova, E.S., Levina, G.V., Naumov, A.D. and Trosnikov, I.V. (2009) The Helical Feature Calculation for the Velocity Field of a Developing Tropical Cyclone. Rus sian Meteorology and Hydrology, 34, 572-580. 
https://doi.org/10.3103/S1068373909090027

[53] Pouquet, A. and Mininni, P.D. (2010) The Interplay between Helicity and Rotation in Turbulence: Implications for Scaling Laws and Small-Scale Dynamics. Philosophical Transactions of the Royal Society A, 368, 1635-1662.

https://doi.org/10.1098/rsta.2009.0284

[54] Marino, R., Baerenzung, J., Mininni, P.D., Pouquet, A., Rorai, C., et al. (2015) Helical Turbulence in Fluids and MHD. In: Fröhlich, J., Kuerten, H., Geurts, B. and Armenio, V., Eds., Direct and Large-Eddy Simulation IX, ERCOFTAC Series, Vol. 20, Springer, Cham, 549-559.

[55] Rotunno, R. (1981) On the Evolution of Thunderstorm Rotation. Monthly Weather Review, 109, 577-586. https://doi.org/10.1175/1520-0493(1981)109<0577:OTEOTR>2.0.CO;2

[56] Davies-Jones, R.P. (1984) Streamwise Vorticity: The Origin of Updraft Rotation in Supercell Storms. Journal of the Atmospheric Sciences, 41, 2991-3006. https://doi.org/10.1175/1520-0469(1984)041<2991:SVTOOU>2.0.CO;2

[57] Lilly, D.K. (1986) The Structure, Energetics, and Propagation of Rotating Convective Storms. Part II: Helicity and Storm Stabilization. Journal of the Atmospheric Sciences, 43, 126-140. https://doi.org/10.1175/1520-0469(1986)043<0126:TSEAPO>2.0.CO;2

[58] Levina, G.V., Zolnikova, N.N. and Mikhailovskaya, L.A. (2017) Cloud-Resolving Numerical Analysis of the Process of Helicity Generation in Conditions of Tropical Cyclogenesis. Sovremennye Problemy Distantsionnogo Zondirovaniya Zemliiz Kosmosa (Current Problems in Remote Sensing of the Earth from Space), 14, 213-222. https://doi.org/10.21046/2070-7401-2017-14-4-213-222

[59] Montgomery, M.T., Nguyen, S.V., Smith, R.K. and Persing, J. (2009) Do Tropical Cyclones Intensify by WISHE? Quarterly Journal of the Royal Meteorological Society, 135, 1697-1714. https://doi.org/10.1002/qj.459

[60] Nolan, D.S., Atlas, R., Bhatia, K.T. and Bucci, L.R. (2013) Development and Validation of a Hurricane Nature Run Using the Joint OSSE Nature Run and the WRF Model. Journal of Advances in Modeling Earth Systems, 5, 382-450.

[61] Wang, Z., Montgomery, M.T. and Dunkerton, T.J. (2009) A Dynamically-Based Method for Forecasting Tropical Cyclogenesis Location in the Atlantic Sector Using Global Model Products. Geophysical Research Letters, 36, L03801. https://doi.org/10.1029/2008GL035586 